\title{
EFFECT OF NON-SURGICAL THERAPY ON SALIVARY NITRIC OXIDE AND LIPID PEROXIDATION LEVELS IN TYPE II DIABETIC AND NON DIABETIC PATIENTS WITH PERIODONTAL DISEASE
}

\author{
NEENA LATHA, ASHITA UPPOOR*, SANGEETA UMESH NAYAK, DILIP G NAIK
}

Department of Periodontology, Manipal College of Dental Sciences, Mangalore - 575 001, Manipal Academy of Higher Education, Karnataka, India. E-mail: ashita.uppoor@manipal.edu

Received: 06 April 2018, Revised and Accepted: 04 May 2018

\section{ABSTRACT}

Objective: Reactive oxygen species have been identified as potential factors causing periodontal tissue destruction. Elevated levels of these in patients with chronic periodontitis and diabetes may aggravate the oxidative stress burden thereby accelerating the tissue damage associated with diabetes. The present study aimed to assess the effect of diabetes and periodontal disease on the oxidative stress markers and the effect of non-surgical therapy on these markers.

Methods: A total of 50 participants were divided into four groups based on the selection criteria. In addition to clinical parameters and biochemical parameters (salivary nitric oxide [NO] and malondialdehyde [MDA] levels) were assessed using spectrophotometric method at baseline and 3 months after non-surgical periodontal therapy.

Result: There was a statistically significant difference in the clinical parameters as well as NO and MDA levels in patients with type 2 diabetes and chronic periodontitis than other three groups at baseline and at 3 months after non- surgical periodontal therapy.

Conclusion: Diabetes mellitus and periodontitis have a compounding effect on the oxidative stress. Periodontal therapy is essential for diabetic patients as it can lower the levels of oxidative damage.

Keywords: Chronic periodontitis, Diabetes mellitus, Malondialdehyde, Salivary nitric oxide, Non-surgical therapy, Oxidative stress.

(c) 2018 The Authors. Published by Innovare Academic Sciences Pvt Ltd. This is an open access article under the CC BY license (http://creativecommons. org/licenses/by/4. 0/) DOIttp://dx.doi.org/10.22159/ajpcr.2018.v11i8.25554

\section{INTRODUCTION}

Periodontitis and diabetes mellitus share a bidirectional interrelationship. Elevated blood glucose levels in hyperglycemia alter the oral microflora. Furthermore, the interaction between advanced glycation end products (AGEs) and their receptors mediate systemic degradation of connective tissue including periodontal tissue. Similarly, periodontitis has been associated with increased insulin resistance due to an increase in serum cytokines such as tumor necrosis factor $\alpha$, interleukin 1 , interleukin 6 , and C reactive proteins [1].

Periodontitis is the sixth complication of diabetes mellitus [2]. Recently reactive oxygen species have been identified as a potential factor causing periodontal tissue destruction [3]. Existing evidence advocates that development of diabetic state may be due to oxidative stress which has occurred due to the underlying pathological condition and may also favor the development of diabetic complications. The stimulated inflammation noticed in the diabetic condition is a direct expression and result of chronic pre-existing oxidative stress [4]

Nitric oxide (NO) is a highly reactive free radical and forms a toxic product, peroxynitrite when combined with superoxide. This has been shown to play a role in both infection and inflammatory response, and hence is considered as a key mediator in the pathogenesis of host destruction [5]. Among the several low molecular weight end products of lipid peroxidation (LPO) malondialdehyde (MDA) are most often measured as an index of peroxidation [6]. The levels of serum and salivary NO and MDA have been found to be increased in periodontitis and diabetes [7-9]. The estimation of salivary NO and MDA can be used as a reliable monitor to assess the oxidative damage in a patient.
As oxidative stress is responsible for the complications of diabetes mellitus, the presence of periodontitis in a diabetic patient may aggravate the oxidative stress burden, thereby accelerating the tissue damage associated with diabetes. Not many studies have assessed the effect of non-surgical therapy on the oxidative stress markers. Thus, this study aimed at assessing the compounding effect of coexisting diabetes and periodontal disease on the oxidative stress markers and the effect of non-surgical therapy on these markers.

\section{METHODS}

The study was conducted in the Department of Periodontology, Manipal College of Dental Sciences, Mangalore. The institutional ethical and review board approved the study protocol. Informed consent was obtained from all the participants before the commencement of the study.

A total of 50 participants ( 30 males and 20 females) above 35 years of age (with a mean age of $50.92 \pm 8.65$ ) reporting to the outpatient section of the department were recruited based on the following criteria.

\section{Inclusion criteria}

The subjects should have at least 20 teeth and have been diagnosed with type 2 diabetes for at least 5 years. They should be on stable dose of insulin/oral hypoglycemic drugs. The subjects should not have undergone any periodontal therapy in the past 6 months or have not been on antibiotics in the past 3 months.

\section{Exclusion criteria}

Pregnant or lactating patients, those on active orthodontic therapy, tobacco users (any form), patients with any other systemic diseases other than type 2 diabetes or subjects with any known complications of diabetes or who require antibiotics management. 
The study participants were grouped into four categories

Group 1 (diabetes with chronic periodontitis): 15 patients with clinical attachment loss of $\geq 5 \mathrm{~mm}$ in at least $30 \%$ [10] of the sites and type II diabetes mellitus at least for the past 5 years under stable doses of insulin/oral hypoglycemic drugs.

Group 2 (systemically healthy chronic periodontitis): 15 systemically healthy subjects with clinical attachment loss of $\geq 5 \mathrm{~mm}$ in at least $30 \%$ of the sites.

Group 3 (diabetes without chronic periodontitis): 10 patients with type II diabetes mellitus with clinically healthy gingiva and no clinical attachment loss.

Group 4 (healthy control): 10 systemically healthy subjects with clinically healthy gingiva and no clinical attachment loss.

For all the patients the following biochemical parameters were assessed:

1. Fasting blood sugar

2. Glycated hemoglobin (HbA1c)

3. Complete lipid profile.

Extraoral and intraoral full-mouth clinical assessment excluding third molars were documented at baseline and 3 months after non-surgical therapy. Plaque index (PI) [11], gingival index (GI) [12], probing pocket depth (PPD), and clinical attachment level (CAL) were recorded as clinical parameters. When the CEJ was masked by a restoration or a crown, the relative CAL was recorded.

\section{Saliva collection and storage}

Subjects were made to sit comfortably in the dental chair with the adequate lightning condition. Patients were asked to rinse their mouth with distilled water. $1 \mathrm{ml}$ of unstimulated whole saliva was collected the day following the clinical examination to avoid contamination with blood (8.30 am-10 am).

The saliva was collected using the drooling method in a $5 \mathrm{ml}$ sterile plastic test tube. The saliva sample was centrifuged at $3000 \mathrm{rpm}$ for $10 \mathrm{~min}$. The supernatant was transferred to a plastic vial and stored at $-70^{\circ} \mathrm{C}$ until the time of assay.

The saliva samples were assessed for NO and LPO end product MDA by spectrophotometric method.

\section{Spectrophotometric assay}

Nitric oxide

Nitrogen monoxide is detected in biological fluids through nitrite. The nitrate present in the sample is reduced to nitrite by reduced nicotinamide adenine dinucleotide phosphate in the presence of the enzyme nitrate reductase.

$$
\text { NR }
$$

Nitrate $+\mathrm{NADPH}+\mathrm{H} \rightarrow$ Nitrite $+\mathrm{NADP}+\mathrm{H}_{2} \mathrm{O}$

The nitrite formed reacts with sulfanilamide (solution A) and N - (1 naphthyl) - ethylene diamine dihydrochloride (solution $\mathrm{B}$ ) to give a red violet diazo dye.
Nitrite + Sulfanilamide + N-(1-naphthyl)-ethylene diamine $\rightarrow$ Diazo dye

The diazo dye is measured on the basis of its absorbance in the visible range at $550 \mathrm{~nm}$.

\section{MDA}

$250 \mu \mathrm{l}$ of saliva was diluted to $500 \mu \mathrm{l}$ with distilled water. To the diluted sample $1 \mathrm{ml}$ of Trichloroacetic acid - thiobarbituric acid - hydrochloric acid reagent was added. The samples were kept in boiling water bath for $15 \mathrm{~min}$. The reaction mixture was cooled and centrifuged. The supernatant was taken, and the optical density of the pink color formed was read at $535 \mathrm{~nm}$. The optical density of the pink color formed was directly proportional to the concentration of MDA in the given sample. The final concentration was expressed in $\mu \mathrm{mol} / \mathrm{l}$.

\section{Non-surgical therapy}

Non-surgical therapy was performed for the patients in Group 1 and Group 2. A pre-procedural rinse of $0.2 \%$ chlorhexidine was advised. Scaling and root planning (SRP) was performed in one session of approximately 2 hours. If SRP could not be completed in one visit, the subjects returned within 48 hours to complete the treatment. Following therapy, patients were instructed on proper oral hygiene measures including $0.2 \%$ chlorhexidine mouth rinse for 2 weeks, appropriate brushing technique, interdental aids, and tongue brushing. The patients were recalled every 2 weeks for follow-up during the 3 months study period.

\section{Statistical analyses}

SPSS (version 11.5, SPSS Inc., India) Statistical Package for the Social Sciences were used for analysis of the data. Mean and standard deviations for age, clinical and biochemical parameters of the subjects in the four groups (Group 1, 2, 3, and 4) were analyzed. The difference among the four groups for all the variables except HbA1c, PPD, and CAL was done using the ANOVA. If the difference was statistically significant further post hoc analysis was performed using Bonferroni test for multiple comparison. HbA1c, PPD, and CAL values were analyzed between two groups using student unpaired t-test. The difference in the variables before and after treatment was assessed using student paired t-test. $\mathrm{p}<0.05$ was considered significant.

\section{RESULTS}

The age range of the study participants ranged from 35 years to 71 years.

The distribution of age and gender was not statistically significant suggesting the groups were well matched.

The clinical parameters of the four groups are summarized in Table 1. Group 1 had a significantly higher plaque score than Group 3 and 4 $(p=0.002)$. The difference between Group 1 and 2 was not statistically significant. Group 3 had higher plaque score than Group $4(\mathrm{p}=0.012)$. There was a statistically significant decrease in the PI score after nonsurgical therapy in both the groups $(\mathrm{p}=0.00)$.

Groups 1 and 2 had significant higher GI score than Groups 3 and 4 $(p=0.00)$. The difference between Groups 1 and 2 was not statistically different $(\mathrm{p}=0.419)$. Group 3 had a statistically significant score than Group 4 ( $\mathrm{p}=0.018)$.

Table 1: An overview of clinical parameters at baseline and 3 months

\begin{tabular}{|c|c|c|c|c|c|c|c|c|c|}
\hline \multirow[t]{2}{*}{ Group } & \multirow[t]{2}{*}{ Age (years) } & \multicolumn{2}{|l|}{ PI } & \multicolumn{2}{|l|}{ GI } & \multicolumn{2}{|l|}{ PPD (mm) } & \multicolumn{2}{|l|}{ CAL (mm) } \\
\hline & & Baseline & 3 months & Baseline & 3 months & Baseline & 3 months & Baseline & 3 months \\
\hline 1 & $51.13 \pm 6.75$ & $1.58 \pm 0.24$ & $0.77 \pm 0.12$ & $1.80 \pm 0.26$ & $1.00 \pm 0.15$ & $6.41 \pm 0.75$ & $4.93 \pm 0.94$ & $7.30 \pm 0.85$ & $5.83 \pm 0.86$ \\
\hline 2 & $48.27 \pm 10.67$ & $1.53 \pm 0.43$ & $0.76 \pm 0.17$ & $1.64 \pm 0.28$ & $0.99 \pm 0.19$ & $5.79 \pm 0.87$ & $4.02 \pm 0.47$ & $6.21 \pm 1.01$ & $4.49 \pm 0.57$ \\
\hline 3 & $56.8 \pm 8.71$ & $1.10 \pm 0.26$ & - & $1.14 \pm 0.26$ & - & - & - & - & - \\
\hline 4 & $48.7 \pm 5.53$ & $0.66 \pm 0.09$ & - & $0.80 \pm 0.08$ & - & - & - & - & - \\
\hline
\end{tabular}

PI: Plaque index, GI: Gingival index, PPD: Probing pocket depth, CAL: Clinical attachment loss 


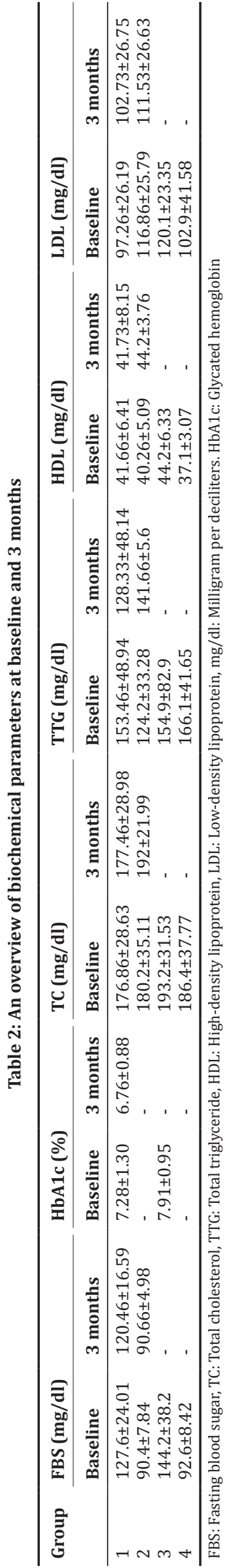

There was a statistically significant decline in the GI score after nonsurgical therapy in both the groups $(\mathrm{p}=0.00)$. The PPD and CAL were recorded in Groups 1 and 2. PPD and CAL were significantly higher in Group 1 compared to Group $2(\mathrm{p}=0.00)$. There was a statistically significant decrease in PPD and CAL in Group 1 at 3 months ( $p=0.001$ and 0.00 , respectively). There was a statistically significant decrease in PPD and CAL in Group 2 at 3 months $(\mathrm{p}=0.000)$. The reduction of CAL was significantly higher in Group 1 than Group $2(\mathrm{p}=0.042)$.

The biochemical parameters of the four groups are summarized in Table 2 .

Group 1 had a significantly higher FBS than Group 2 and Group 3 ( $\mathrm{p}=0.000,0.002$, respectively). The difference between Group 1 and Group 3 was not statistically significant $(\mathrm{p}=0.442)$. Group 3 had a statistically higher FBS than Groups 2 and $4(p=0.000)$. The difference between Groups 2 and 4 was nonsignificant $(p=1.000)$. There was a statistically significant reduction in FBS value in Group $1(\mathrm{p}=0.034)$ whereas in Group 2 the difference was nonsignificant (0.901). Group 1 showed a significantly higher reduction in FBS than Group $2(\mathrm{p}=0.042)$.

The difference in the HbA1c values between Group 1 and Group 3 was nonsignificant $(p=0.208)$. There was a statistically significant reduction in the HbA1c value in Group 1 at 3 months ( $p=0.008)$.

There was no statistically significant difference in the total cholesterol value among the four groups $(p=0.64)$. The mean total triglyceride value of Groups 1, 2, 3, and 4 not statistically significant $(\mathrm{p}=0.218)$. There was a decrease in the TTG value in Group 1 which was not statistically significant $(\mathrm{p}=0.927)$. There was an increase in the TTG value in Group 2 which was also not statistically significant $(\mathrm{p}=0.053)$.

The difference in HDL value among the four groups was statistically significant $(\mathrm{p}=0.042)$. Further, post hoc analysis showed that Group 3 had a statistically higher HDL than Group $4(\mathrm{p}=0.035)$. The difference among the other groups was nonsignificant. There was a slight increase in the HDL value in both the groups at 3 months which were not statistically significant. The difference in LDL value among the four groups was nonsignificant $(\mathrm{p}=0.163)$.

The NO and MDA levels at baseline and 3 months are summarized in Table 3. The NO value in Group 1 was significantly higher than Groups 2,3 , and $4(p=0.000)$. Group 2 had a significantly higher NO value than Groups 3 and 4 ( $p=0.006,0.000$, respectively). The difference between Groups 3 and 4 was nonsignificant ( $\mathrm{p}=1.000)$. There was a statistically significant reduction in NO in both the groups $(\mathrm{p}=0.003,0.001)$.

Group 1 had a significantly higher MDA value than Group $4(\mathrm{p}=0.035)$ whereas the difference between Group 1, Group 2, and Group 3 was nonsignificant $(p=1.000,0.360$, respectively). Group 2 had a significantly higher MDA value than Group $4(\mathrm{p}=0.032)$. The difference between Group 2 and 3, 3 and 4 was nonsignificant ( $p=0.336,1.000)$.

The correlation between clinical and biochemical parameters was done using Karl Pearson's correlation test (Table 4).

NO was positively correlated with PPD and CAL at baseline which was statistically significant $(r=0.709, p=0.003$ and $r=0.846, p=0.000$, respectively). At 3 months NO was positively correlated with PPD and CAL $(\mathrm{r}=0.436, \mathrm{p}=0.104$ and $\mathrm{r}=0.542, \mathrm{p}=0.037)$. The correlation was statistically significant only between NO and CAL.

In Group 2, NO was positively correlated with PPD and CAL at baseline and at 3 months $(r=0.714, p=0.003, r=0.638, p=0.010, r=0.449, p=0.093$, $\mathrm{r}=0.403, \mathrm{p}=0.136$, respectively). The correlation was statistically significant between NO and PPD, CAL at baseline.

\section{DISCUSSION}

Diabetes mellitus (DM) which is one of the public health issues is a metabolic disorder characterized by the presence of chronic 
hyperglycemia accompanied by an impairment of carbohydrate, lipid, and protein metabolism that can lead to premature death [13].

In a previous study [9], it was demonstrated that serum NO and LPO are elevated in diabetes mellitus whereas in another study [14] an inverse relationship between NO and MDA was found. These ROS are associated with the microvascular complications of diabetes. It is also known that severe periodontal disease can lead to endothelial dysfunction which is an early event in atherogenesis [15-19]. In this study, the compounding effect of coexisting diabetes and periodontitis on the levels of oxidative stress markers was assessed.

The clinical parameters PI, GI, PPD, and clinical attachment loss were assessed and the result was in accordance with earlier studies [18]. There was a significantly higher PPD and CAL in Group 1 when compared to Group 2. Type 2 diabetes may be considered best as a modifier of host-parasite interactions in the periodontium.[19,20] This modification may encourage the virulent expression of pathogens in the subgingival ecology in a direct fashion and/or an alteration of the host's inflammatory/immune response to these microorganisms. The net result is increased severity of periodontitis in subjects with diabetes.

Contrary to many other studies [21-23], there was no statistically significant difference in the FBS and HbA1c value among Group 1 and 3. This can be attributed to the presence of predominantly controlled diabetic patients in this study. In this study, though the duration of diabetes was more in Group 3 than Group 1, the absence of periodontitis is Group 3 might be attributed to the good oral hygiene and decreased PI, GI values in this group. This is in agreement with the study by Tervonen and Oliver [24] who reported that duration of diabetes did not have a significant effect on the severity of periodontitis.

In Group 1, the total triglyceride value was higher than normal whereas the other lipid parameters values were in the normal limit [25]. In Group 2, except LDL other lipid parameters were within the normal range [26]. In periodontitis, changes in lipid profile are thought to be mediated by cytokines, which may be produced at the inflamed periodontal tissues in high quantities [27-29]. In diabetes, hyperglycemia occurs due to the inability of tissues to utilize the glucose. The mobilization of the fatty acid from adipose tissue occurs for energy purpose, and extra fatty acid will be stored in liver, which is converted into triglycerides [30]. There was no statistically significant difference in the lipid profile among Groups 1,2, 3, and 4.

In the present study, whole unstimulated saliva was collected as a sample. It represents the oral condition and also has some

Table 3: An overview of NO and MDA at baseline and 3 months

\begin{tabular}{llllll}
\hline \multirow{2}{*}{ Group } & NO & & & MDA \\
\cline { 2 - 3 } \cline { 5 - 6 } & Baseline & 3 months & & Baseline & 3 months \\
\hline 1 & $9.08 \pm 2.33$ & $7.28 \pm 0.86$ & & $2.17 \pm 0.52$ & $1.19 \pm 0.50$ \\
2 & $4.15 \pm 1.68$ & $3.24 \pm 1.23$ & & $2.19 \pm 1.62$ & $1.39 \pm 1$ \\
3 & $1.76 \pm 1.02$ & - & & $1.33 \pm 1.12$ & - \\
4 & $0.84 \pm 0.24$ & - & $0.91 \pm 0.21$ & - \\
\hline
\end{tabular}

NO: Nitric oxide, MDA: Malondialdehyde elements of gingival crevicular fluid (GCF) and tissue metabolites that may be helpful in the tissue degradation. In addition, stimulated salivary flow has been proved to increase saliva volume and disrupt the concentration [31-33]. There are evidences that saliva has considerable antioxidant capacity, and oxidative stress may happen as a consequence of LPO, and impaired capacity of saliva antioxidant power. Saliva collection is simpler, easier and non-invasive and can be a good alternative for blood identification and prognosis of many diseases.

NO is a central mediator of host response and is an important agent in the pathogenesis of host destruction. NO synthesis and iNOS activity are stated to be increased in periodontal tissues. Activity of NO system in diabetes is controversial, with few studies demonstrating higher level [34,35], a low level [36,37], or no change [38-40].

In the present study, elevated NO levels were found in Group 1 and Group 2 when compared to the Group 3 and Group 4. This result is in agreement with various other studies $[5,7,41]$. In periodontitis, the elevated NO is due to the increased expression of iNOS by the PMNs, macrophages, and endothelial cells. The higher NO production is a mirror image of an immune-activated state wherein iNOS have been upregulated by inflammatory cytokines and other mediators. The level of NO was more in Group 1 when compared to Group 2. This is in agreement to the study by Pan et al. [5] in which the authors found no higher iNOS positivity and iNOS expressing cells in tissue biopsy specimens between patients with diabetes and chronic periodontitis and nondiabetics with periodontitis.

MDA is the major and commonly studied product of polyunsaturated fatty acid peroxidation that is shown to rise following oxidative stress. An increased LPO level following periodontitis has been noted in many studies $[6,8,42]$. In addition to oxygen-derived free radicals, glycated collagen has also been shown to raise the oxidative breakdown of lipids compared to normal collagen which causes increased MDA in serum and tissues of diabetic subjects [24]. In the present study, we found a significant rise in the level of LPO in Group 1 and Group 2 in comparison with healthy controls (Group 4). This result is in agreement with other studies $[8,42,45]$. In the study by Tsai et al. [8], increased concentration of LPO was found in GCF and whole saliva. The mechanism of superoxide anion production during the interaction of periodontal pathogens or their products and neutrophils within periodontal tissues or pocket may increase the GCF concentration. The increased concentration in the whole saliva may be because of the elevated percentage of GCF in saliva in periodontitis. The higher MDA level in the saliva in periodontitis patients may also be due to increased leakage of ROS to saliva from serum and GCF. LPO caused by oxygen radicals from Fusobacterium-stimulated neutrophils has also been suggested as a possible model for the emergence of periodontitis [46]. In diabetic patients hypertriglyceridemia is associated with oxidation modification of LDL, protein glycation, and auto-oxidation thus leading to the elevated production of LPO. Hence, the elevated serum triglycerides in the diabetic patients might have had a role in the increased production of MDA in this study [47].

In our study, the MDA level of Groups 1, 2, and 3 was significantly higher than Group 4. This is in agreement with the study by Wei et al. [47] who found a significant difference in salivary MDA between chronic

Table 4: Correlation between clinical and biochemical parameters using Pearson's correlation

\begin{tabular}{|c|c|c|c|c|c|c|c|c|}
\hline \multirow[t]{3}{*}{ Parameter } & \multicolumn{4}{|l|}{ Group A } & \multicolumn{4}{|l|}{ Group B } \\
\hline & \multicolumn{2}{|c|}{ Baseline } & \multicolumn{2}{|c|}{3 months } & \multicolumn{2}{|c|}{ Baseline } & \multicolumn{2}{|c|}{3 months } \\
\hline & r value & p value & r value & $p$ value & r value & p value & r value & p value \\
\hline NO - PPD & 0.709 & $0.003^{*}$ & 0.436 & 0.104 & 0.714 & $0.003^{*}$ & 0.449 & 0.093 \\
\hline NO - CAL & 0.846 & $0.000^{*}$ & 0.542 & $0.037^{*}$ & 0.638 & $0.010^{*}$ & 0.403 & 0.136 \\
\hline
\end{tabular}


periodontitis and control group whereas a study by Sonoki et al. [48] demonstrated an elevated LPO level in patients with diabetes and periodontitis when compared with periodontitis. LPO was measured by an alternative method which indicates an earlier step in LPO. The lack of difference between Group 1 and 2 might be explained by the fact that though MDA levels are increased by LPO during tissue destruction, no correlation was found between the degree of inflammation and MDA levels in many studies [30,49].

Non-surgical therapy was performed for patients in Group 1 and 2. In our study, non-surgical therapy was performed over 2 days as no significant difference in clinical and metabolic parameters were found using full mouth disinfection in type II diabetic patients [50].

Three months following non-surgical therapy, there was a statistically significant reduction in PI, GI, PPD, and CAL in both Groups 1 and 2. This result is in agreement with various other studies [51,52]. This result may be due to the fact that patients with well-controlled diabetes mellitus might respond to non-surgical therapy similarly well as healthy controls [48] and many of the patients in this study were controlled, diabetic patients.

There was a statistically significant reduction in the FBS and HbA1c values $(7.28 \pm 1.30$ to $6.76 \pm 0.88)$ in Group 1 following non-surgical therapy. There was no significant difference in FBS value in Group 2. This is in agreement with a study by Grossi et al. [49] and Kiran et al. [52] who concluded that elimination of periodontal infection and improvement of periodontal inflammation significantly reduces the level of HbA1c in the short term, hence, improving diabetes metabolic control. Stewart et al. [51] reported a reduction in HbA1c value from 9.5\% to $7.6 \% 10$ months following non-surgical therapy. A systematic review in 2010 concluded that periodontal therapy for type II diabetic patients is favorable and can reduce the HbA1c levels [53,54]. Two of four poorly controlled diabetic patients in the group improved to moderate control. The role of non-surgical therapy on the reduction in $\mathrm{HbA1c}$ level could not be determined as various other factors such as patients' diet, exercise, hypoglycemic drugs, and increased awareness during the study period (Hawthorne effect) might also have had an effect. Adequate knowledge of the individual person with diabetes is considered to be a cornerstone to become competent in selfmanagement and, in turn, prevent further health complications [54].

Following non-surgical therapy, no significant difference was found in the lipid profile in the two groups. In Group A, there was a reduction in TTG but a slight increase in TC, HDL, and LDL which was not statistically significant. In Group 2, there was a slight increase in TC, TTG, and HDL a decrease in LDL though the results were not statistically significant. This result is in agreement with other studies in which the authors reported no significant difference in the lipid profile after non-surgical therapy $[26,55]$.

Three months following non-surgical therapy there was a statistically significant reduction in the levels of NO in both Groups 1 and 2. This result is in agreement with the study by Gullu et al. [56] who reported a reduction in the iNOS expression and number of inflammatory cells 2 months after non-surgical therapy. There was also an increase in the enzyme arginase activity following scaling and root planing. Arginase might originate from oral bacteria or host cells or both sources. Arginase can downregulate NO production by decreasing intracellular arginine concentrations. Hence, there exists an inverse relationship between iNOS and arginase, i.e. arginase might prevent the sustained overproduction of NO.

Although there was a reduction in the level of NO in both Groups 1 and 2, the level did not reach baseline control value. In the study by Gullu et al. [56], the authors reported that modified Widman flap surgery was more effective in lowering the count of iNOS expressing cells compared to SRP. Since all the patients in our study had moderate to severe periodontitis, non-surgical therapy was not very effective in reducing the level of NO.
There was a statistically significant reduction to control level in the level of MDA in both Groups 1 and 2. This result is in agreement with another study by Tsai et al. [8] who reported a significant reduction in salivary LPO concentration 1 month after non-surgical therapy. Wei et al. [47] found a statistically significant reduction of MDA level to the control level 4 months after non-surgical therapy. The significant decrease of MDA after therapy suggests that the total antioxidant capacity in chronic periodontitis appears to be restored to control level by successful nonsurgical therapy. Sonoki et al. [48] suggested that periodontal therapy presumably might have an antioxidant effect equal to those of antioxidant therapies, such as vitamin $\mathrm{E}$ administration in type 2 diabetic patients.

The limitations of this study are that the HbA1c value of patients in Group 2 and Group 3 (at 3 months) was not assessed which would have helped in predicting the effect of non-surgical therapy on glycemic control. Confounding factors such as alcohol consumption, diet, stress, and genetic factors could not be controlled. The abnormal lipid profile of some of the patients might also have had a role in the elevated LPO levels. In a recent study [57], it was reported that gingival tissue biopsy was more appropriate for estimating oxidative stress as gingiva is in close proximity to the periodontal tissues where the onset of periodontal disease happens. In the current work, the effect of nonsurgical therapy was assessed after 3 months which might be too short a duration to define the effect of therapy on these markers.

To the best of our knowledge, this is one among very few studies to compare the salivary levels of NO and MDA in diabetic patients with periodontitis. It was found that there was elevated oxidative stress independent of the glycemic control in diabetic patients which decreased after periodontal therapy. This stresses the importance of periodontal health in diabetic patients.

Although there was a substantial reduction in NO and MDA levels in both the groups following non-surgical therapy, the difference was not statistically significant between the two groups. This can be attributed to the fact that saliva sample represents a more localized oral environment whereas diabetes is a generalized chronic inflammatory condition. Hence, the elevated oxidative stress in the diabetes mellitus and periodontitis group was more due to periodontitis than diabetes [5].

\section{CONCLUSION}

In this study, a free radical, $\mathrm{NO}$ and an end product of LPO, namely MDA were assessed in diabetic and non-diabetic patients with periodontitis. Furthermore, the effect of non-surgical therapy on the levels of these markers was also assessed. It was found that the levels of these markers were significantly higher in diabetic patients with periodontitis than periodontitis and non-diabetic patients. Non-surgical therapy resulted in a substantial reduction in the levels of these markers.

Diabetes mellitus and periodontitis have a compounding effect on the oxidative stress. Periodontal therapy is essential for diabetic patients as it can lower the levels of oxidative damage thereby it may prevent the microvascular complications associated with diabetes. Also combining non-surgical therapy with host modulation agents such as sub-antimicrobial dose doxycycline may offer an additional benefit in diabetic patients. Treatment with antioxidants and iNOS inhibitors such as mercaptoethylguanidine along with hypoglycemic should be the future of diabetic therapy.

Future research should focus on the establishment of the exact role of NO and MDA in the pathogenesis of periodontal disease in diabetic patients. Studies involving surgical therapy with a longer duration of follow-up should be planned to evaluate the effect of periodontal therapy on these markers.

\section{ACKNOWLEDGMENT}

Nil. 


\section{AUTHOR'S CONTRIBUTIONS}

Neena Latha conducted the study and prepared the manuscript. Dr. AshitaUppor and Dr.Dilip G Naik guided her to design the study and to conduct the study. Dr. Sangeeta has helped her conduct the study and prepare the manuscript.

\section{CONFLICTS OF INTEREST}

The authors confirm that this article content has no conflicts of interest.

\section{REFERENCES}

1. Taiyeb-Ali TB, Cheta Raman RP, Vaithialingam RD. Relationship between periodontal disease and diabetes mellitus: An Asian perspective. Periodontol 2000 2011;56:258-68.

2. Loe H. Periodontal disease. The sixth complication of diabetes mellitus. Diabetes Care 1993;16:329-34.

3. Chapple IL, Matthews JB. The role of reactive oxygen and antioxidant species in periodontal tissue destruction. Periodontol 2000 2007;43:160-32.

4. Allen EM, Matthews JB, Connor RO, O'Halloran D, Chapple IL. Periodontitis and type II diabetes: Is oxidative stress the mechanistic link? Scott Med J 2009;54:41-7.

5. Pan Z, Guzeldemir E, Toygar HU, Bal N, Bulut S. Nitric oxide synthase in gingival tissues of patients with chronic periodontitis and with and without diabetes. J Periodontal 2010;81:109-20.

6. Khalili J, Biloklytska HF. Salivary malondialdehyde levels in clinically healthy and periodontal diseased individuals. Oral Dis 2008;14:754-60.

7. Reher VG, Zenobio EG, Costa FO, Reher P, Soares RV. Nitric oxide levels in saliva increase with severity of periodontitis. J Oral Sci 2007:49:271-6

8. Tsai CC, Chen HS, Chen SL, Ho YP, Ho KY, Wu YM, et al. Lipid peroxidation: A possible role in the production and progression of chronic periodontitis. J Periodontal Res 2005;40:378-84.

9. Aydin A, Orhan H, Sayal A, Özata M, Sahin G, Issımer A. Oxidative stress and nitric oxide related parameters in Type II diabetes mellitus: Effects of glycemic control. Clin Biochem 2001;34:65-70

10. Halliwell B. Reactive oxygen species in living systems: Source, biochemistry and role in human diseases. Am J Med 1991;91:14S-22S.

11. Brennan PA, Thomas GJ, Langdon JD. The role if nitric oxide in oral diseases. Arch Oral Biol 2003:48:93-100.

12. Lohinai Z, Benedek P, Feher E, Györfi A, Rosivall L, Fazekas Á, et al. Protective effects of mercaptoethylguanidine, a selective inhibitor of inducible nitric oxide synthase, in ligature-induced periodontitis in the rat. Br J Pharmacol 1998;123:353-60

13. Deepa J, Aleykutty NA, Jyoti H. Effect of combination of two plant extracts on diabetes mellitus. Int J Pharm Pharm Sci 2018;10:49-52.

14. Lohinai Z, Stachlewitz R, Virag L, Székely AD, Haskó G, Szabó C. Evidence for reactive nitrogen species formation in the gingivomucosal tissue. J Dent Res 2001;80:470-5.

15. Daghigh F, Borghaei RC, Thornton RD, Bee JH. Human gingival fibroblasts produce nitric oxide in response to pro inflammatory cytokines. J Periodontol 2002;73:392-400.

16. Sosroseno W, Barid I, Herminajeng E, Susilowati H. Nitric oxide production by a murine macrophage cell line (RAW264.7) stimulated with lipopolysaccharide from Actinobacillusactinomycetemcomitans. Oral Microbiol Immunol 2002;17:72-8

17. Kim SJ, Ha MS, Choi EY, Choi JI, Choi IS. Prevotella intermedia lipopolysaccharide stimulates release of nitric oxide by inducing expression of inducible nitric oxide synthase. J Periodont Res 2004;39:424-1

18. Matejka M, Partyka L, Ulm C, Solar P, Sinzlnger H. Nitric oxide synthesis is increased in periodontal disease. J Periodont Res 1998;33:517-8

19. Kendall HK, Haase HR, Li H, Xiao Y, Bartold PM. Nitric oxide synthase type-II is synthesized by human gingival tissue and cultured human gingival fibroblasts. J Periodont Res 2000;35:194-200.

20. Lappin DF, Kjeldsen M, Sander L, Kinane DF. Inducible nitric oxide synthase expression in periodontitis. J Periodont Res 2000;35:369-73.

21. Hirose M, Ishihara K, Saito A, Nakagawa T, Yamada S, Okuda K. Expression of cytokines and inducible nitric oxide synthase in inflamed gingival tissue. J Periodontol 2001;72:590-7.

22. Batista A, Silva TA, Chun JH, Lara VS. Nitric oxide synthesis and severity of human periodontal disease. Oral Dis 2002;8:254-60.

23. Bartold PM, Wiebkin OW, Thonard JC. The effect of oxygen-derived free radicals on gingival proteoglycans and hyaluronic acid. J Periodont
Res 1984;19:390-400

24. Tervonen T, Oliver RC. Long-term control of diabetes mellitus and periodontitis. J Clin Periodontol 1993;20:431-5.

25. Girotti AW. Mechanisms of lipid peroxidation. J Free Rad Biol Med $1985 ; 1: 87-95$

26. Varani J, Fligiel SEG, Till GO, Kunkel RG, Ryan US, Ward PA. Pulmonary endothelial cell killing by human neutrophils: Possible involvement of hydroxyl radical. Lab Invest 1985;53:656-63.

27. Liberman TO, Baltimore O. Activation of interleukin-6 gene expression through NF-kB transcription factor. Mol Cell Biol 1990;22:2327-34

28. Poli G, Parola M. Oxidative damage and fibrogenesis. Free Rad Biol Med 1997;22:287-305

29. Hemnani T, Parihar MS. Reactive oxygen species and oxidative DNA damage. Indian J Physiol Pharmacol 1998;42:440-52.

30. Del Rio D, Stewart AJ, Pellegrini N. A review of recent studies on malondialdehyde as toxic molecule and biological marker of oxidative stress. Nutr Metab Cardiovas Dis 2005;15:316-8.

31. Tricković-Janjić O, Cvetković T, Apostolović M. Analysis of enzyme activity and the level of malondialdehyde in the saliva of children with gingivitis. Vojnosanit Pregl 2009;66:892-6.

32. Akalm FA, Baltacioglu E, Alver A, Karabulut E. Lipid peroxidation levels and total oxidant status in serum, saliva and gingival crevicular fluid in patients with chronic periodontitis. J Clin Periodontol 2007;34:558-65.

33. Brownlee M. Biochemistry and molecular cell biology of diabetic complications. Nature 2001;414:813-20.

34. Green K, Brand, MD, Murphy MP. Prevention of mitochondrial oxidative damage as a therapeutic strategy in diabetes. Diabetes Care 2004;53:s110-7.

35. Mohanty P, Hanouda W, Garg R, Aljada A, Ghanim H, Dandona P. Glucose challenge stimulates reactive oxygen species (ROS) generation by leucocytes. J Clin Endo Metab 2000;85:2970-3.

36. Quagliaro L, Piconi L, Assaloni R, Martinelli L, Motz E, Ceriello A. Intermittent high glucose enhances apoptosis related to oxidative stress in human umbilical vein endothelial cells: The role of Protein Kinase C and NAD(P)HOxidase activation. Diabetes 2003;52:2795-04

37. Gillery P, Monboisse JC, Maquart FX, Borel JP. Glycation of proteins as a source of superoxide. Diabet Metab 1998;14:25-30

38. Freita JP, Filipe PM, Guerra Rodrigo F. Lipid peroxidation in type 2 normolipidemic diabetic patients. Diabetes Res Clin Pract $1997 ; 36: 71-5$

39. Skaleric U, Gaspirc B, McCartney-Francis N, Masera A, Wahl SM. Proinflammatory and antimicrobial nitric oxide in gingival fluid of diabetic patients with periodontal disease. Infect Immun 2006:74:7010-3.

40. Al-Rawi NH. Oxidative stress, antioxidant status and lipid profile in the saliva of type 2 diabetes. Diab Vasc Dis Res 2011;8:22-8

41. Grossi SG, Genco RJ. Periodontal disease and diabetes mellitus: A twoway relationship. Ann Periodontol 1998;3:51-61.

42. Fredriksson MI, Gustaffson AK, Bergstrom KG, Asman BE. Constitutionally hyperreactive neutrophils in periodontitis. J Periodontol 2003;74:219-24

43. Brock GR, Matthews JB, Butterworth CJ, Chapple IL. Local and systemic antioxidant capacity in periodontitis and health. J Clin Periodontol 2004;31:515-21.

44. Iacopino AM. Diabetic periodontitis: A possible lipid-induced defect in tissue repair through alteration of macrophage phenotypes and function. Oral Dis 1995:1:214-29.

45. Lee TI, Sun MF, Wen YR, Kuo PL, Liu CF. Elevated nitric oxide levels inhibit malondialdehyde production in patients with diabetes mellitus in Taiwan. Nutr Metab Cardiovas Dis 2005;15:452-3.

46. Miller LS, Manwell MA, Newbold D, Reding ME, Rasheed A, Blodgett $\mathrm{J}$, et al. The relationship between reduction in periodontal inflammation and diabetes control: A report of 9 cases. J Periodontol 1992;63:843-8

47. Wei D, Zhang XL, Wang YZ, Yang CX, Chen G. Lipid peroxidation levels, total oxidant status and superoxide dismutase in serum, saliva and gingival crevicular fluid in chronic periodontitis patients before and after periodontal therapy. Aust Dent J 2010;55:70-8.

48. Sonoki K, Nakashima S, Takata Y, Naito T, Fujisawa K, Ootsubo T, et al. Decreased lipid peroxidation following periodontal therapy in type 2 diabetic patients. J Periodontol 2006;77:1907-13

49. Grossi SG, Skrepcinski FB, DeCaro T, Robertson DC, Ho AW, Dunford RG, et al. Treatment of periodontal disease in diabetes reduces glycated hemoglobin. J Periodontol 1997;68:713-9.

50. Christgau M, Palitzsch KD, Schmalz G, Kreiner U, Frenzel S. Healing response to non-surgical periodontal therapy in patients with diabetes 
mellitus: Clinical, microbiological and immunologic results. J Clin Periodontol 1998;25:112-24.

51. Stewart JE, Wager KA, Friedlander AH, Zadeh HH. The effect of periodontal treatment on glycemic control in patients with type 2 diabetes mellitus. J Clin Periodontol 2001;28:306-10.

52. Kiran M, Arpak N, Unsal E, Erdoğan MF. The effect of improved periodontal health on metabolic control in type 2 diabetes mellitus. J Clin Periodontol 2005;32:266-72.

53. Rodrigues DC, TabaJr M, NovaesJr AB, Souza SL, Grisi MF. Effect of non-surgical periodontal therapy on glycemic control in patients with type 2 diabetes mellitus. J Periodontol 2003;74:1361-7.

54. Teeuw WJ, Gerdes VE, Loos BG. Effect of periodontal treatment on glycemic control of diabetic patients: A systematic review and metaanalysis. Diabetes Care 2010;33:421-7.

55. Dussa KN, Sahay RK, Sundararajan P, Ardhanarao MV. Adaptation of diabetes knowledge questionnaire for south Asian countries context. Int J Pharm Pharm Sci 2017;9:252-6.

56. Gullu C, Ozmeric N, Tokman B, Elgün S, Balos K. Effectiveness of scaling and root planning versus modified Widman flap on nitric oxide synthase and arginase activity in patients with chronic periodontitis. J Periodont Res 2005;40:168-75.

57. Ozer L, Elgun S, Ozdemir B, Pervane B, Ozmeric N. Arginine-nitric oxide-polyamine metabolism in periodontal disease. J Periodontol 2011;82:320-8. 